\title{
Cell Broadening Revisited: Results from High-Resolution Large-Eddy Simulations of Cold Air Outbreaks
}

\author{
M. SCHRÖTER, S. RAASCH, AND H. JANSEN \\ Institut für Meteorologie und Klimatologie, Universität Hannover, Hannover, Germany
}

(Manuscript received 13 May 2004, in final form 3 November 2004)

\begin{abstract}
Large-eddy simulations (LES) have been carried out in order to investigate the structure and development of organized mesoscale cellular convection (MCC) during cold air outbreaks (CAOB) and to reevaluate results by Müller and Chlond. Some limitations of this and other earlier LES studies of CAOBs have been removed by using a parallelized model with both a large horizontal domain and a fine grid resolution. These model simulations reveal a hitherto undiscovered insight into the development of MCC.

It is shown that MCC with aspect ratios larger than 10 only develop in the presence of diabatic heat sources, that is, latent heat release within the clouds and cloud-top radiative cooling, which confirms results from previous studies. Simulated cells are of closed type. The wind field is seen to be correlated with the liquid water field, and thus, dynamic variables are organized on the mesoscale as well. Updrafts predominantly occur in regions with high liquid water content. Two-dimensional spectral analysis confirms clear peaks at the wavelength of the MCC. The dynamic variables are characterized by an organized conglomeration of randomly distributed up- and downdrafts, where each convective cell can hardly be detected by eye. Whereas scalar variables like temperature and the liquid water path field are characterized by large scales with aspect ratios of 9 to 11, in the vertical velocity field multiple scales are present simultaneously, with small scales having aspect ratios between 1 and 3 contributing most to the total energy, but also large scales correlated with scales seen in the thermodynamic variables.
\end{abstract}

\section{Introduction}

During so-called cold air outbreaks (CAOB), initially cloud free and stably stratified air is advected from land or from an ice surface over a warmer (sea) surface. Subsequent heating and moistening from below causes the development of a convective boundary layer, in which a typical sequence of convection patterns can be observed: As soon as the cold air encounters the warm sea surface, roll-like secondary flow patterns develop, appearing in satellite images as so-called cloud streets (Etling and Brown 1993; Atkinson and Zhang 1996). Further downstream, the roll pattern gradually changes to a hexagonal cellular convection pattern that can contain either open or closed cells. Open cells consist of broad central areas of descending air, enclosed by relatively narrow rings of updrafts, whereas closed cells are characterized by reversed circulation. Near the ice edge or coast, the convection patterns define aspect ratios, the quotient of cell

Corresponding author address: M. Schröter, Alfred-WegenerInstitut für Polar-und Meeresforschung, Am Handelshafen 12, D-27570 Bremerhaven, Germany.

E-mail: mschroeter@awi.bremerhaven.de diameter and boundary layer height, ranging from 1 to 3 . Subsequently, they gradually increase up to the scale of mesoscale cellular convection, reaching typical values for the aspect ratios of 10 to 30 . The frequent occurrence of so-called mesoscale cellular convection patterns (MCC) over vast regions of the oceans has been revealed by satellite imaging and its properties have been reviewed in detail by various authors (Agee 1984; Etling and Brown 1993; Atkinson and Zhang 1996). Within the last decade both CAOBs and MCCs have been studied intensively using numerical simulation (Fiedler 1993; Fiedler and Khairoutdinov 1994; Müller and Chlond 1996; Dörnbrack 1997; Jonker et al. 1999). Most of these studies yielded diabatic processes to be an essential prerequisite for cell broadening and thus large aspect ratios as observed in nature.

Müller and Chlond (1996, hereafter MC96) studied a CAOB situation as observed during the ARKTIS 1991 experiment. Latent heat release due to condensation and especially radiative cooling at the cloud top were identified as processes leading to cell broadening. To bypass the computer memory problem, they introduced a regridding technique. The boundary layer development was simulated by using large-eddy simulation (LES) in combination with a method of dynamically 
adapting domain size and resolution to the evolving structures. The horizontal size of their model domain and the horizontal resolution of the computational grid was continually adjusted to the scale of the dominant convective structures during the course of the simulations. At the end of their runs, the horizontal domain extended over $100 \times 100 \mathrm{~km}^{2}$ but with a horizontal grid spacing of $1.6 \mathrm{~km}$.

Thus, some restrictions remain due to insufficient computer resources available at the time. An LES of MCC during a CAOB situation needs a computational domain covering a horizontal area on the order of $\sim 100$ $\mathrm{km} \times 100 \mathrm{~km}$ to allow the development of large-scale convective cells. Simultaneously a grid resolution of $\sim 50$ to $100 \mathrm{~m}$ is necessary to resolve smaller energy containing eddies of the convective boundary layer (CBL), which might interact with larger cells. Under these circumstances, the numerical grid requires $\sim 10^{8}$ grid points. In the study of Dörnbrack (1997), who used LES to investigate the influence of both heating at the lower cloud boundary by condensation and cloud-top radiative cooling on the broadening of convective cells, the horizontal extent was not sufficient to follow the subsequent evolution of cell broadening toward a stationary state. At the end of his LES, one convective cell filled out about one-half of the box side and thus the domain size became too small to allow for further growth of the cells. In the study of MC96, the anisotropic numerical grid required the use of artificially increased horizontal diffusion coefficients, which limits the resolution of the smaller scales and thus also the effect small scales may have on large-scale structures. Additionally, large anisotropic eddy diffusion coefficients may affect the aspect ratio of the simulated cells (Ray 1965).

The purpose of this note is to remove all these uncertainties by means of LES using simultaneously a model domain that is large enough to capture several mesoscale structures, and a high grid resolution to explicitly resolve small-scale interactions.

\section{Simulation setup and initial conditions}

The parallelized LES model (PALM) applied in the present study is specially designed for use on massively parallel computers. It is described in detail in Raasch and Schröter (2001) and has been applied successfully to the atmospheric and oceanic boundary layer (e.g., Weinbrecht and Raasch 2001; Noh et al. 2003; Letzel and Raasch 2003; Kanda et al. 2004).

We consider a CAOB situation observed during the ARKTIS 1991 experiment (Brümmer 1992, 1999). This setting was already used as reference state by MC96, where a more detailed description of the experimental setup is given. The initialization parameters for the simulations performed here were directly adopted from MC96 and can be described as follows: At a latitude of $72^{\circ} \mathrm{N}$, an initially dry and stably stratified air mass is advected by a geostrophic wind of $22 \mathrm{~m} \mathrm{~s}^{-1}$ from the pack ice across the relatively warm water of ice-free ocean. The model domain covers an area of $70.4 \mathrm{~km} \times$ $70.4 \mathrm{~km}$ using a grid spacing of $100 \mathrm{~m}$ in the horizontal and approximately $5.5 \mathrm{~km}$ in the vertical direction with a mesh size of $50 \mathrm{~m}$ (for $z>3.3 \mathrm{~km}$, the vertical grid spacing is smoothly stretched) resulting in $705 \times 705 \times$ $80 \approx 4 \times 10^{7}$ grid points. Based on these initial conditions we have performed two runs. The first run (hereafter referred to as WET) includes moist processes like cloud formation, whereas the second run (hereafter referred to as DRY) treats the moisture as a passive scalar only, cloud formation and radiation processes have been switched off. In all model simulations, the initial wind profile for the three-dimensional simulation was obtained by a one-dimensional version of PALM using prescribed temperature and humidity/scalar profiles $\left(\theta_{z=0, t<0}=261.741 \mathrm{~K}, \partial \theta / \partial z=5.18 \mathrm{~K} \mathrm{~km}^{-1}, q_{z=0, t<0}=\right.$ $\left.1.5137 \mathrm{~g} \mathrm{~kg}^{-1}, \partial q / \partial z=-0.273 \mathrm{~g} \mathrm{~kg}^{-1} \mathrm{~km}^{-1}\right)$. With the beginning of the three-dimensional simulation, the surface temperature was set to its value observed near the ice edge $\left(\theta_{z=0, t=0}=273 \mathrm{~K}\right)$ and was increased linearly further on according to observations during ARKTIS $1991\left(\partial \theta / \partial t=1.32 \times 10^{-4} \mathrm{~K} \mathrm{~s}^{-1}\right)$. At the surface, moisture saturation has been assumed (initially, $q_{z=0, t=0}=$ $3.7 \mathrm{~g} \mathrm{~kg}^{-1}$ ). Surface fluxes have been calculated via the Monin-Obukhov similarity. To initialize a turbulent state of the boundary layer flow, the velocity field was perturbed by imposing small random perturbations. All simulations performed for this study cover a period of 12.5 h. Table 1 shows an overview of some initial parameters as well as resulting characteristic flow parameters.

\section{Results}

We first present results of the WET model simulation. In general, the evolution of the simulated mean flow state is typically for cloudy CBLs (e.g., Brümmer et al. 1986; Cuijpers and Duynkerke 1993; Brümmer 1999). Figure 1 shows the temporal development of vertical mean profiles of various variables. Because of the increasing surface temperature, an unstably stratified layer near the surface is initiated and leads to the development of a monotonically growing convective boundary layer. The surface buoyancy fluxes decrease slightly, from 0.23 to $0.15 \mathrm{~K} \mathrm{~m} \mathrm{~s}^{-1}$, whereas the latent heat fluxes increase from 0.04 to $0.08 \mathrm{~g} \mathrm{~kg}^{-1} \mathrm{~m} \mathrm{~s}^{-1}$ at the end of the simulation (see Fig. 1 and Table 1). After about $1.5 \mathrm{~h}$, the flow reaches a quasi-stationary state recognizable by the well-mixed profiles of the horizontal wind speed and the potential temperature, as well as by the temporal self-similarity of the vertical profiles of the second moments (fluxes and variances). At the end of the simulation after $12.5 \mathrm{~h}$, the boundary layer depth reaches a value of approximately $3200 \mathrm{~m}$, while the cloud layer ranges over more than $2000 \mathrm{~m}$. 
TABLE 1. List of initial parameters for all simulations; $L_{x}, L_{y}$, $L_{z}$ : domain size in $x, y$ direction; $d x, d y, d z$ : grid spacings; $\left(u_{g}, v_{g}\right)$ : components of the geostrophic wind; $f$ : the Coriolis parameter; $F \downarrow z_{T}$ is the impinging infrared radiative flux on the top of the domain; $z_{0}, z_{\theta}, z_{q}$ are the roughness lengths for impulse, temperature, and moisture, respectively. The subscript 0 indicates the value of the respective parameter at the surface, $\Delta$ the increase at the beginning of the three-dimensional simulation, $\partial / \partial z$ the initial value of the vertical gradient. For DRY $q$ represents a passive scalar.

\begin{tabular}{|c|c|c|c|}
\hline Parameter & WET & DRY & Units \\
\hline \multicolumn{4}{|l|}{$\begin{array}{l}\text { Boundary conditions/ } \\
\text { initialization parameters }\end{array}$} \\
\hline Cloud physics & Yes & No & \\
\hline Radiation & Yes & No & \\
\hline Passive scalar & No & Yes & \\
\hline$L_{x}, L_{y}$ & \multicolumn{2}{|c|}{70.4} & $\mathrm{~km}$ \\
\hline$L_{z}$ & \multicolumn{2}{|c|}{5.5} & $\mathrm{~km}$ \\
\hline$d x, d y$ & \multicolumn{2}{|c|}{100.0} & $\mathrm{~m}$ \\
\hline$d z$ & \multirow{2}{*}{\multicolumn{2}{|c|}{$\begin{array}{l}50.0 \\
(0,22)\end{array}$}} & $\mathrm{m}$ \\
\hline$\left(u_{g}, v_{g}\right)$ & & & $\mathrm{m} \mathrm{s}^{-1}$ \\
\hline$f^{3}$ & \multicolumn{2}{|c|}{1.387} & $10^{-4} \mathrm{~s}^{-1}$ \\
\hline$\theta_{0, t<0}$ & \multirow{2}{*}{\multicolumn{2}{|c|}{$\begin{array}{c}261.741 \\
5.18\end{array}$}} & $\mathrm{~K}$ \\
\hline$\partial \theta / \partial z$ & & & $\mathrm{~K} \mathrm{~km}^{-1}$ \\
\hline$\Delta \theta$ & \multicolumn{2}{|c|}{11.259} & $\mathrm{~K}$ \\
\hline$\overline{\partial \theta} / \partial t_{0}$ & \multicolumn{2}{|c|}{1.32} & $10^{-4} \mathrm{~K} \mathrm{~s}^{-1}$ \\
\hline$q_{0, t<0}$ & \multirow{2}{*}{\multicolumn{2}{|c|}{$\begin{aligned} & 1.5137 \\
&-0.273\end{aligned}$}} & $\mathrm{~g} \mathrm{~kg}^{-1}$ \\
\hline$\partial q / \partial z$ & & & $\mathrm{~g} \mathrm{~kg}^{-1} \mathrm{~km}^{-1}$ \\
\hline$\Delta q$ & \multicolumn{2}{|c|}{2.1863} & $\mathrm{~g} \mathrm{~kg}^{-1}$ \\
\hline$z_{0}$ & \multicolumn{2}{|c|}{5} & $10^{-4} \mathrm{~m}$ \\
\hline$z_{\theta}$ & \multicolumn{2}{|c|}{1} & $10^{-4} \mathrm{~m}$ \\
\hline$z_{q}$ & \multicolumn{2}{|c|}{1} & $10^{-6} \mathrm{~m}$ \\
\hline$F \downarrow z_{T}$ & 80 & - & $\mathrm{W} \mathrm{m}^{-2}$ \\
\hline Resulting parameters & & & \\
\hline$z_{i}(t=12.5 \mathrm{~h})$ & 3200.0 & 2200.0 & $\mathrm{~m}$ \\
\hline$u^{*}$ & 0.75 & 0.75 & $\mathrm{~m} \mathrm{~s}^{-1}$ \\
\hline$w^{*}$ & 2.5 & 2.4 & $\mathrm{~m} \mathrm{~s}^{-1}$ \\
\hline$\left\langle w^{\prime \prime} \theta^{\prime \prime}\right\rangle_{0, t=12.5 \mathrm{~h}}$ & 0.14 & 0.187 & $\mathrm{~K} \mathrm{~m} \mathrm{~s}^{-1}$ \\
\hline$\left\langle w^{\prime \prime} \theta_{v}^{\prime \prime}\right\rangle_{0, t=12.5 \mathrm{~h}}$ & 0.154 & - & $\mathrm{K} \mathrm{m} \mathrm{s}^{-1}$ \\
\hline$\left\langle w^{\prime \prime} q^{\prime \prime}\right\rangle_{0, t=12.5 \mathrm{~h}}$ & 0.079 & 0.061 & $\mathrm{~g} \mathrm{~kg}^{-1} \mathrm{~m} \mathrm{~s}^{-1}$ \\
\hline
\end{tabular}

Because of the release of latent heat in the cloud layer, the turbulence is enhanced leading to an increase of the velocity variances and the buoyancy flux in this region. The buoyancy flux decreases linearly with height from the surface up to the cloud base, where it reaches a negative value. It then increases upward and reaches a local maximum at the top of the lower third of the cloud layer. This maximum is caused by release of latent heat and radiative heating at the cloud base. These features of the buoyancy flux profile give strong evidence of decoupling of the cloud-topped boundary layer. In general, decoupling is associated with negative buoyancy fluxes below the cloud base and a cloud-base local minimum of $\overline{w^{\prime 2}}$ (e.g., Turton and Nicholls 1987; Bretherton and Wyant 1997). In WET, decoupling occurs for $t \geq 7 \mathrm{~h}$ at about $z=0.4 z_{i}$. Following Turton and Nicholls (1987), the surface mixed layer lies below that level and the decoupled mixed layer above. Without describing the results of DRY in detail at this point, an evidence of decoupling could not be observed in DRY. As summarized by Stevens (2000), a major feature of decoupling is that the tendency toward a two- layer stratocumulus-topped boundary layer is the first phase of the climatologically important stratocumulus to trade-cumulus transition. However, we do not assume decoupling to be the major reason for the differences between WET and DRY model results (see later in this paper).

After reaching the quasi steady state, the organization of the secondary flow is characterized by closed cellular convection. The pattern is best detectable in the thermodynamic field variables, such as the liquid water path (LWP) field (LWP $=\int_{0}^{z_{\max }} q_{l} d z$ ). To obtain a more realistic view of the cloud pattern comparable to the view from a satellite, Fig. 2 shows contour plots of cloud reflectivities obtained from the LWP field.

As is typical for closed cellular convection, broad areas of large values of reflectivities (light areas) in the center of the cells are enclosed by narrow ring-shape areas of low values of reflectivities (dark areas). Here, large amounts of LWP correspond to high values of reflectivities and vice versa. The cellular pattern could also be identified in the $w$ field, shown in Fig. 3 (top), because of a positive correlation with the $q_{l}$ field. In areas of small liquid water content, predominantly downdrafts can be identified whereas in the center of the cells, updrafts are more frequent than downdrafts. Accordingly, each mesoscale convection cell occurs in the vertical velocity field as an organized conglomeration of randomly distributed up- and downdrafts.

To study the cell broadening, a spectral analysis was performed. Figure 4 shows the variance spectra of LWP and the vertical velocity as a function of the aspect ratio, which is defined here as the ratio of wavelength to boundary layer height. The dominant wavelength may be interpreted as a measure of the typical diameter or the typical size of coherent structures occurring in the specific variable field. Spectra were calculated as in MC96 from two-dimensional Fourier analysis of horizontal cross sections located approximately in the middle of the developing cloud layer. Fourier coefficients were averaged along circles in order to generate a one-dimensional presentation. The $w$ spectra were calculated in the horizontal plane, in which the resolved variance of the vertical velocity reaches a local maximum. Since spectra were multiplied by the wavenumber, a peak in the spectrum can be interpreted as an important contribution to the variance of the variable.

The broadening of convective cells can be clearly identified by the shift of the dominant spectral peaks toward larger wavenumbers in the spectra of the LWP with increasing time. Table 2 gives an overview of the temporal evolution of the diameter and the aspect ratio A of dominant cell structures estimated by spectral analysis. The dominant wavelength in the LWP field increases monotonically from $\sim 6.4 \mathrm{~km}$ after 2 -h simulated time to $\sim 35.3 \mathrm{~km}$ at the end of the simulation.

In accordance with the increase of the dominant wavelength, the dominant aspect ratio of the LWP field 

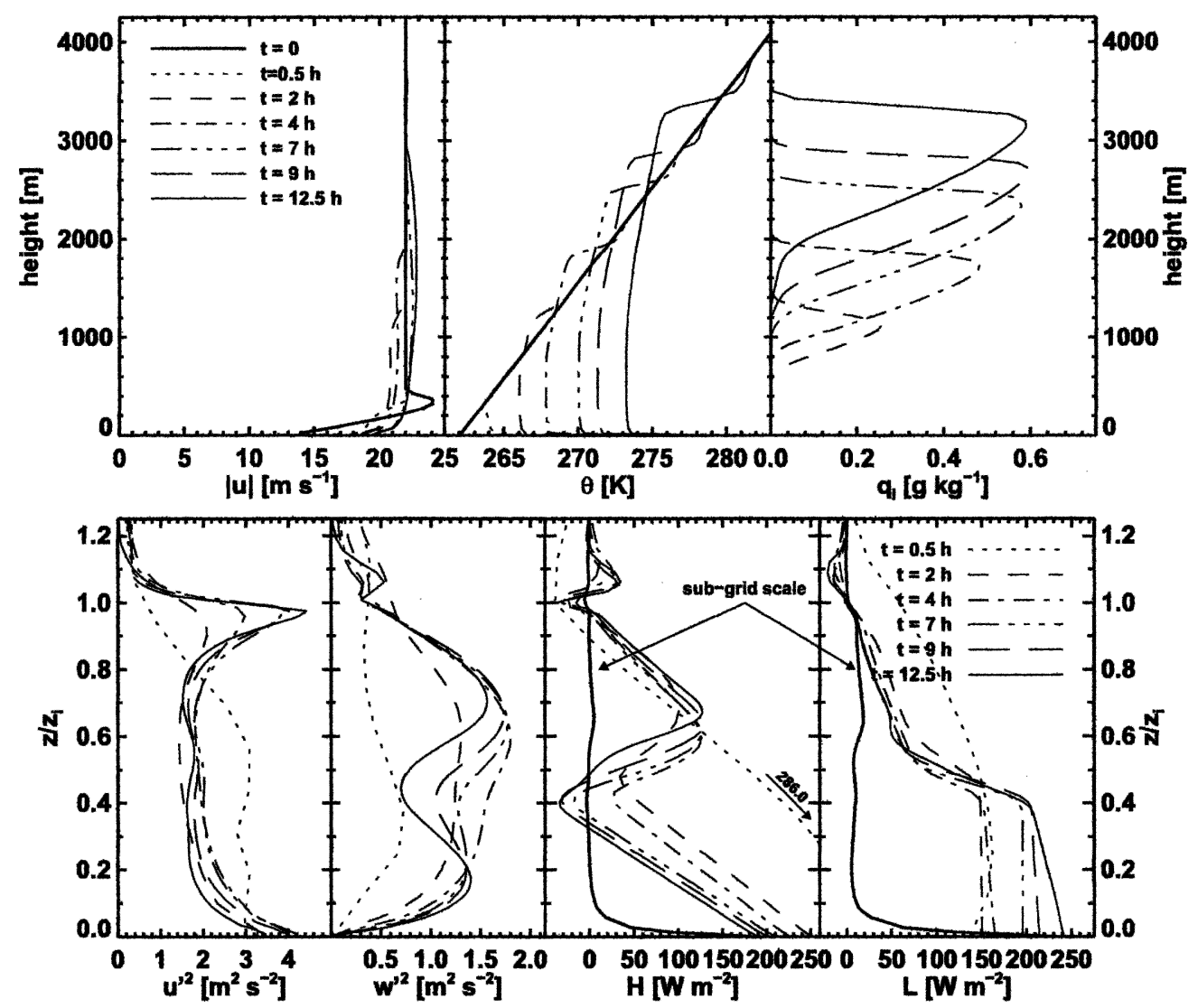

FIG. 1. Vertical mean profiles of various variables at different time levels. (top, from left to right) Mean wind speed, liquid water potential temperature, liquid water content. (bottom, from left to right) Horizontal and vertical velocity variances, sensible and latent heat fluxes. For the sensible and latent heat fluxes, typical subgrid parts of the total fluxes are additionally shown. To calculate the heat fluxes, a density of $1.225 \mathrm{~kg}$ $\mathrm{m}^{-3}$ was assumed.

(cf. Fig. 4) also increases nearly monotonically (apart from a deviation within the period from $t=7 \mathrm{~h}$ to $t=$ $9 \mathrm{~h}$ ) from $A \approx 5$ at $t=2 \mathrm{~h}$ to $A \approx 11$ at $t=12.5-\mathrm{h}$ simulated time. In contrast to the LWP field, the vertical velocity field is not dominated by such large scales. The scales with the largest contribution to the variance of the vertical velocity have aspect ratios between 1 and 4 as typical for convective boundary layers (Hardy and Ottersten 1969). However, because of the positive correlation between the liquid water field and the vertical velocity field, each dominant scale in the LWP field appears as a corresponding local peak in the spectra of the vertical velocity. Hence, the spectral density of the vertical velocity at large aspect ratios increases with increasing time. Whereas at $t=2 \mathrm{~h}$, scales with wavelengths larger than $10 \mathrm{~km}$ practically are not present, later on, a significant part of the variance of the vertical velocity field can be attributed to such large scales.

To point out the correlation between the dynamics of the flow and its thermodynamics (temperature and liquid water content), Fig. 5 presents autocorrelation functions of the vertical velocity $[\rho(w)]$ and the potential temperature $[\rho(\theta)]$ as well as cross-correlation func- tions of $w$ and $q_{l}\left[\rho\left(w, q_{l}\right)\right]$ and $\theta$ and $q_{l}\left[\rho\left(\theta, q_{l}\right)\right]$, all as functions of the separation distance $r$. Whereas $\rho(w)$ drops to zero in a very short spatial lag nearly independently of time and height, $\rho(\theta)$ shows an increase with time and height. Consequently, the vertical velocity field is characterized by an irregular small-scaled signal typical for turbulent processes, whereas the temperature and the liquid water field (as seen before) are dominated by larger scales. The positive correlation between $w$ and the thermodynamic variables is, even at small lags, less than the correlation between the thermodynamic variables among themselves. Thus, considering the whole model domain, updrafts are somewhat correlated with positive temperature fluctuations (defined as deviations from the horizontal means) and analogous downdrafts are correlated with negative temperature fluctuations, whereas high liquid water content and positive temperature fluctuations (as low liquid water content and negative temperature fluctuations) display high correlations even at large distances. This is connected to the fact that $w$ itself is characterized by multiple small structures, while $\theta$ and $q_{l}$ are dominated by large scales. 

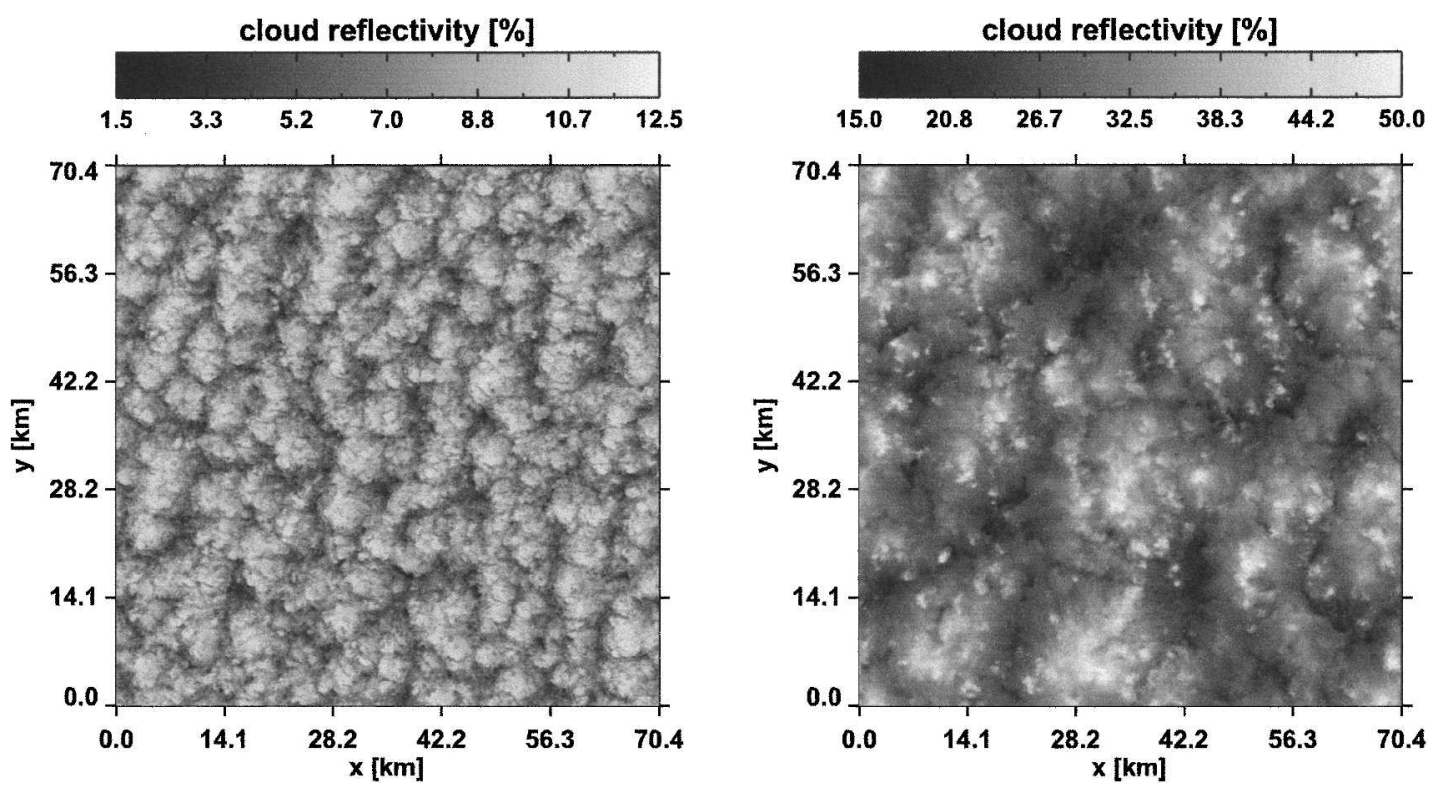

FIG. 2. Horizontal cross sections of cloud reflectivities calculated from the liquid water path (WET simulation): (left) $t=4 \mathrm{~h}, z=1600 \mathrm{~m}$; (right) $t=12.5 \mathrm{~h}, z=3100 \mathrm{~m}$. Cloud reflectivities have been estimated using the parameterization of Slingo (1989) assuming an equivalent radius of the drop size distribution of $6 \mu \mathrm{m}$ and a solar zenith angle of $60^{\circ}$.

We now turn to the second run (DRY), which was performed using the same initial conditions as WET, but without consideration of moist processes. Here, moisture is treated as a passive scalar. In general, it is found that in contrast to WET, the formation of MCC fails to appear in DRY. Accordingly, neither increasing aspect ratios nor increasing spectral energy levels at larger scales are observed in either scalar or dynamic variables. Figure 6 shows the power spectra of the vertical velocity and the scalar field in the middle of the boundary layer height at different time levels as a function of the aspect ratio. The spectra of the scalar field are independent of height (not shown). Thus, Fig. 6 is representative for all model heights and may be compared with Fig. 4.

Throughout the simulation, the dominant aspect ratios remain nearly constant in both fields. The dominant scales of the vertical velocity are slightly smaller than those of the passive scalar and range between 1.6 and 2.9, as is typical for cloudless convective boundary layers (Hardy and Ottersten 1969), whereas the dominant scales of the passive scalar remain between 2.6 and 4.7 (cf. Table 2). Figure 7 shows horizontal cross sections of the vertical velocity and the passive scalar field at the end of the simulation. Up- and downdrafts are not part of a large-scale organized cell structure as in WET. Rather, individual up- and downdrafts appear as a cell-like pattern. This cellular pattern seems to be slightly stretched in the direction of the mean wind from south to north. The passive scalar field is characterized by broader structures compared to the vertical velocity field. However, the structures occurring here are not similar to the large cell structures observed in the case of MCC.

\section{Discussion and conclusions}

Opening the capability of today's supercomputers by using a parallelized model we have been able to perform an LES study of a CAOB situation with both a large horizontal domain and a fine grid resolution for the first time. This work emphasizes the important role that parallel computing technology plays in our ability to study the evolution of organized convection in the atmosphere. Some limitations of earlier LES studies of CAOBs, especially those of MC96, have been removed.

Both our study and MC96 agree on major results; however, there are some differences between our investigations and those of MC96 that are attributable to the regridding technique used by MC96. Analyzing the data of the current high-resolution study, we are able to get much more detailed insight into the structure of MCC. Whereas the convective pattern occurring in the course of WET appears as closed convective cells, MC96 could not clearly classify the convection pattern occurring in their model simulation as closed or open. This might be a result of the coarse grid they had to use, which also led to the simulated fields being much smoother, including reduced minima and maxima of the vertical velocity, in comparison to our WET simulation. Figure 3 shows a comparison of horizontal cross sections through the $w$ field in our simulation $(t=12.5$ $\mathrm{h}$, right) and MC96's basic control run ( $t=15 \mathrm{~h}$, lower graph). The $w$ field simulated in WET is characterized 
(a)
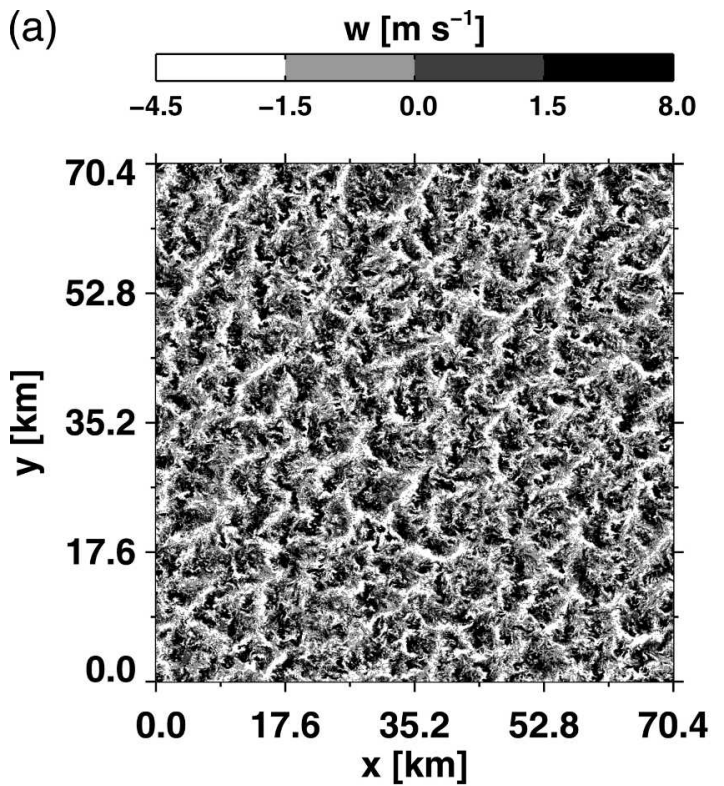

(b)
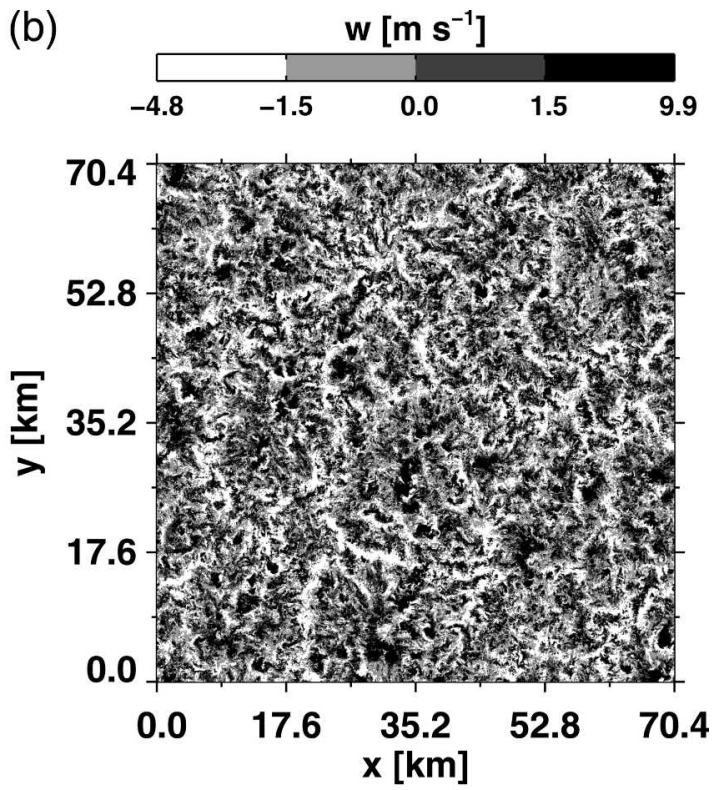

(c)

$\mathbf{w}\left[\mathrm{m} \mathrm{s}^{-1}\right]$
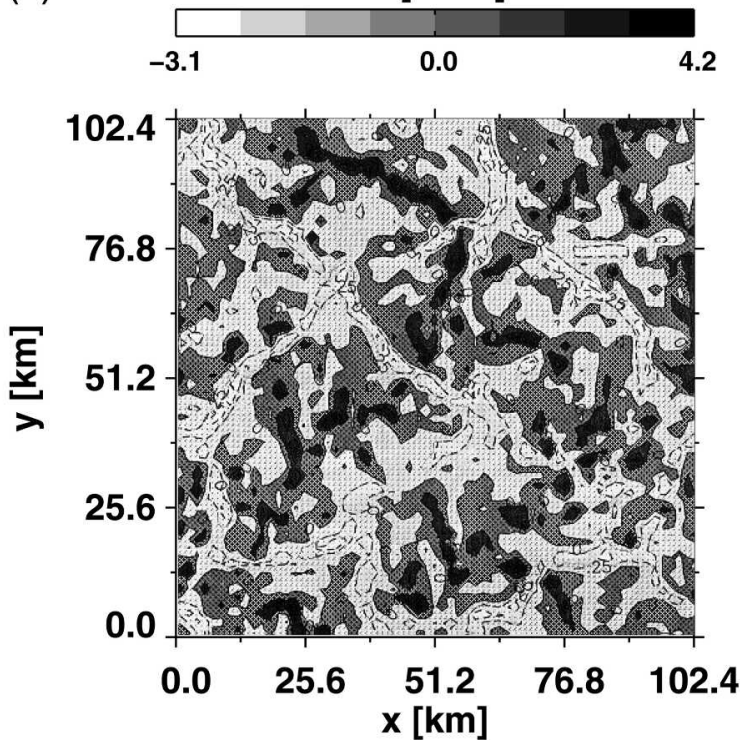

FIG. 3. (top) Horizontal cross sections of vertical velocity $w$ (WET simulation): (left) $t=4 \mathrm{~h}, z=1300 \mathrm{~m}$; (right) $t=12.5 \mathrm{~h}, z=2150 \mathrm{~m}$. The cross sections are located at the level where the resolved variance of the vertical velocity reaches a local maximum. (bottom) Result of MC96's basic control run for $t=15 \mathrm{~h}$, discussed in section 4 [figure courtesy Müller and Chlond (1996)].

by an organized conglomeration of randomly distributed up- and downdrafts, where each convective cell can hardly be detected by eye. In contrast, the $w$ field simulated by MC96 is characterized by much broader regions of coherent up- and downdrafts.

Unlike MC96, who observed increasing dominant aspect ratios in the liquid water field as well as in the vertical velocity field, in WET only the liquid water field shows an increasing dominant scale, whereas in the $w$ field, the magnitude of any dominant aspect ratio decreases with time at the expense of several distinct peaks. Thus, structures at the mesoscale exist, but are not dominating, as smaller structures are evident too. This discrepancy is another effect that we attribute to the regridding technique used by MC96. For example, at the end of the simulation of MC96, only scales with wavelength larger than $3200 \mathrm{~m}$ were explicitly resolved by the numerical grid. With regard to the corresponding spectra (Fig. 4), the left part of the spectrum would not be existent. From this technical aspect, scales with aspect ratios $A>1$ are affected, which excludes the LWP since no peaks exist at the smaller scales. As far as 


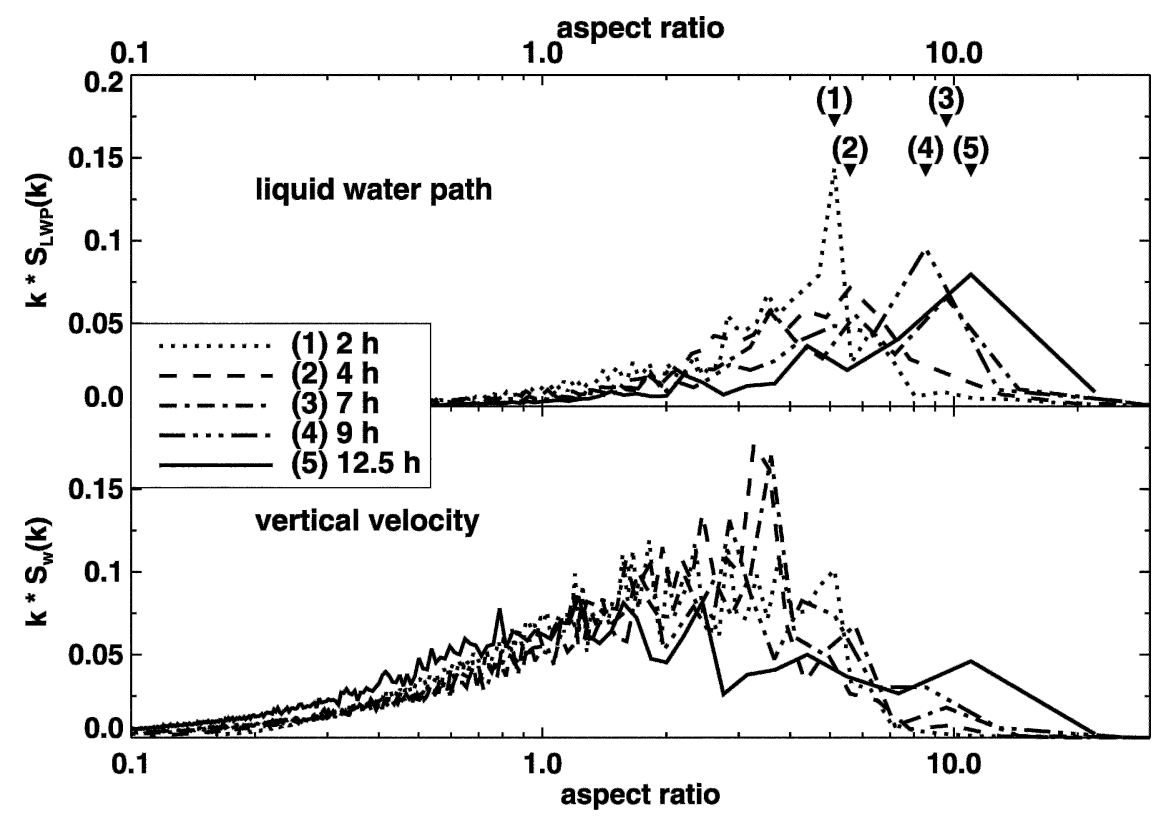

FIG. 4. (top) Power spectra of the liquid water content and (bottom) vertical velocity, WET simulation. Aspect ratios according to dominant scales at specific time levels are marked by triangles.

the vertical velocity field is concerned, the cutoff at $\lambda$ $\sim 3200 \mathrm{~m}$ does affect numerous structures on the small scale. Furthermore, because of additional filtering effects of the subgrid scheme (Moeng and Wyngaard 1988) the spectral density of scales of the internal subrange would have been reduced. Thus, large scales are able to have a larger contribution to the total variance compared to a simulation with a higher resolution.

In general, our results are in good agreement to the earlier studies of MC96. It seems that one has to strictly distinguish between two different kinds of cell broadening. Whereas on the scale of single coherent (e.g., upand downdrafts) turbulent structures only the scalar variables show increasing aspect ratios, on the scale of MCC both dynamic and scalar field variables broaden because of a positive correlation between the thermodynamics and dynamics of the turbulent flow. In both studies, a clear MCC signal is observed only when considering longwave radiative processes and latent heat release due to condensation processes (WET). Hence, we substantiate the statement that additional diabatic heat is responsible for the existence and broadening of MCC, which is furthermore supported by recent findings of de Roode et al. (2004). Mesoscale fluctuations do occur under boundary conditions, which do not necessarily have to include additional diabatic heat sources, but these fluctuations are reaching the scale of MCC only in the presence of additional diabatic heat sources.

With regard to the study of Jonker et al. (1999), which has been improved recently by de Roode et al. (2004), we have found some discrepancies in DRY con- cerning the coupling of scale growing and the entrainment to the surface flux ratio. It has been found that mesoscale fluctuations are negligibly small for conserved variables that have an entrainment to surface flux ratio of about -0.25 (de Roode et al. 2004). In case of the potential temperature, the results of DRY agree well with this finding. The flux ratio is about -0.15 for all time levels and no enlargement of scales has been

TABLE 2. Diameters $\lambda$ and aspect ratios $A$ of the dominant cell structures in the liquid water field for different time levels estimated by spectral analysis.

\begin{tabular}{|c|c|c|c|c|c|c|}
\hline Run & Time (h) & 2 & 4 & 7 & 9 & 12.5 \\
\hline \multirow[t]{7}{*}{ WET } & $z_{i}(\mathrm{~m})$ & 1250 & 1800 & 2450 & 2750 & 3200 \\
\hline & \multicolumn{6}{|c|}{ LWP power $_{\max }$} \\
\hline & $\lambda(\mathrm{km})$ & 6.4 & 10.1 & 23.5 & 23.5 & 35.3 \\
\hline & $A$ & 5.1 & 5.6 & 9.6 & 8.6 & 11.0 \\
\hline & \multicolumn{6}{|c|}{$w$ power $_{\max }$} \\
\hline & $\lambda(\mathrm{km})$ & 2.3 & 5.9 & 8.8 & 7.8 & 3.9 \\
\hline & $A$ & 1.8 & 3.3 & 3.6 & 2.8 & 1.2 \\
\hline \multirow[t]{7}{*}{ DRY } & $z_{i}(\mathrm{~m})$ & 900 & 1250 & 1600 & 1800 & 2200 \\
\hline & \multicolumn{6}{|c|}{$s$ power $_{\max }$} \\
\hline & $\lambda(\mathrm{km})$ & 3.7 & 5.9 & 4.1 & 6.4 & 10.1 \\
\hline & $A$ & 4.1 & 4.7 & 2.6 & 3.6 & 4.6 \\
\hline & \multicolumn{6}{|c|}{$w$ power $_{\max }$} \\
\hline & $\lambda(\mathrm{km})$ & 1.5 & 3.7 & 3.7 & 5.0 & 5.0 \\
\hline & $A$ & 1.7 & 3.0 & 2.3 & 2.8 & 2.3 \\
\hline
\end{tabular}



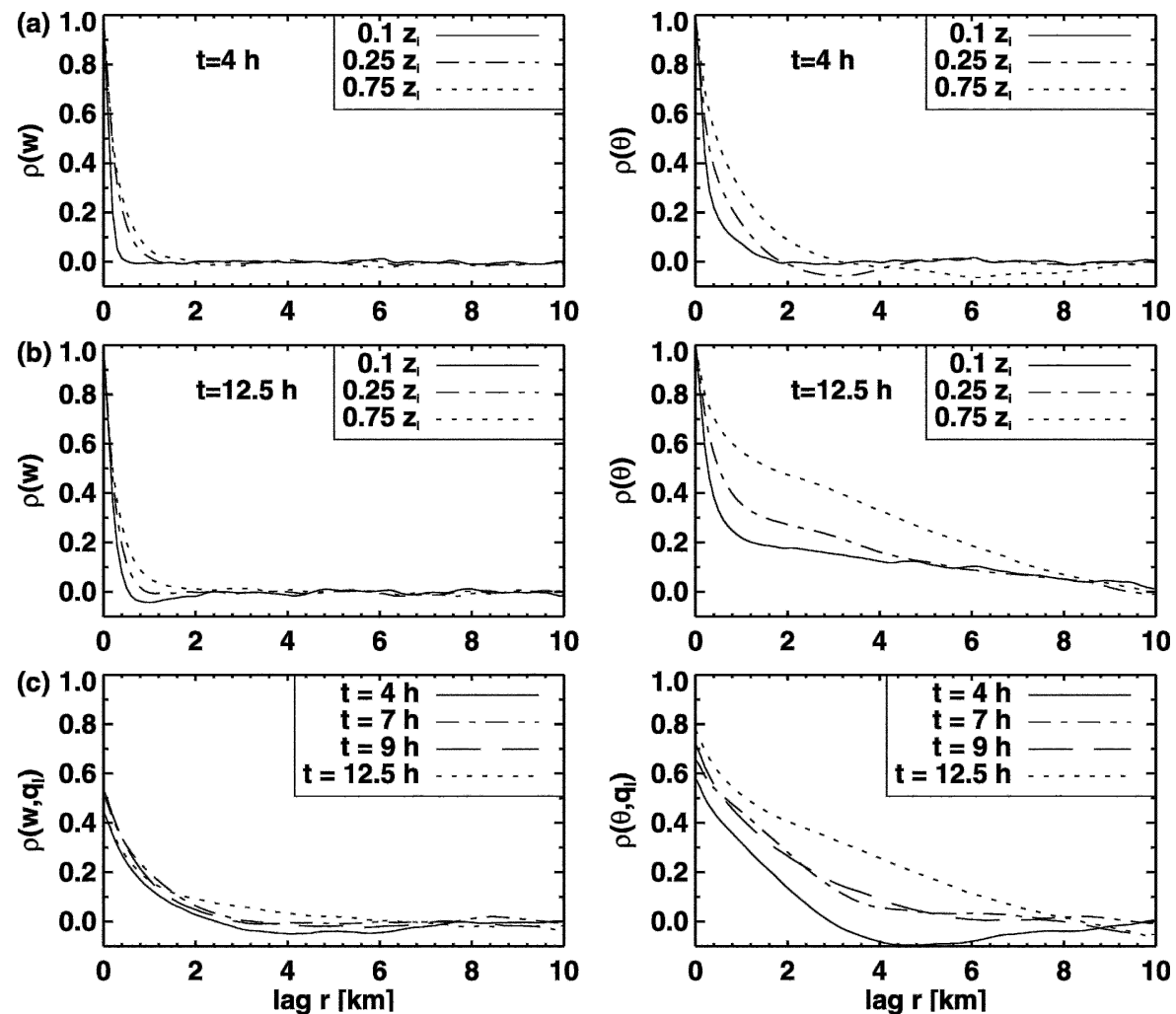

FIG. 5. Autocorrelation functions (left) $\rho(w)$ and (right) $\rho(\theta)$ as functions of the separation distance $r$ for (top) $t=4 \mathrm{~h}$ and (middle) $t=12.5 \mathrm{~h}$ and at different height levels (cf. legend). Cross-correlation functions (bottom left) $\rho\left(w, q_{l}\right)$ and (bottom right) $\rho\left(\theta, q_{l}\right)$ as functions of the separation distance $r$ for $z=0.75 z_{i}$ at different time levels.

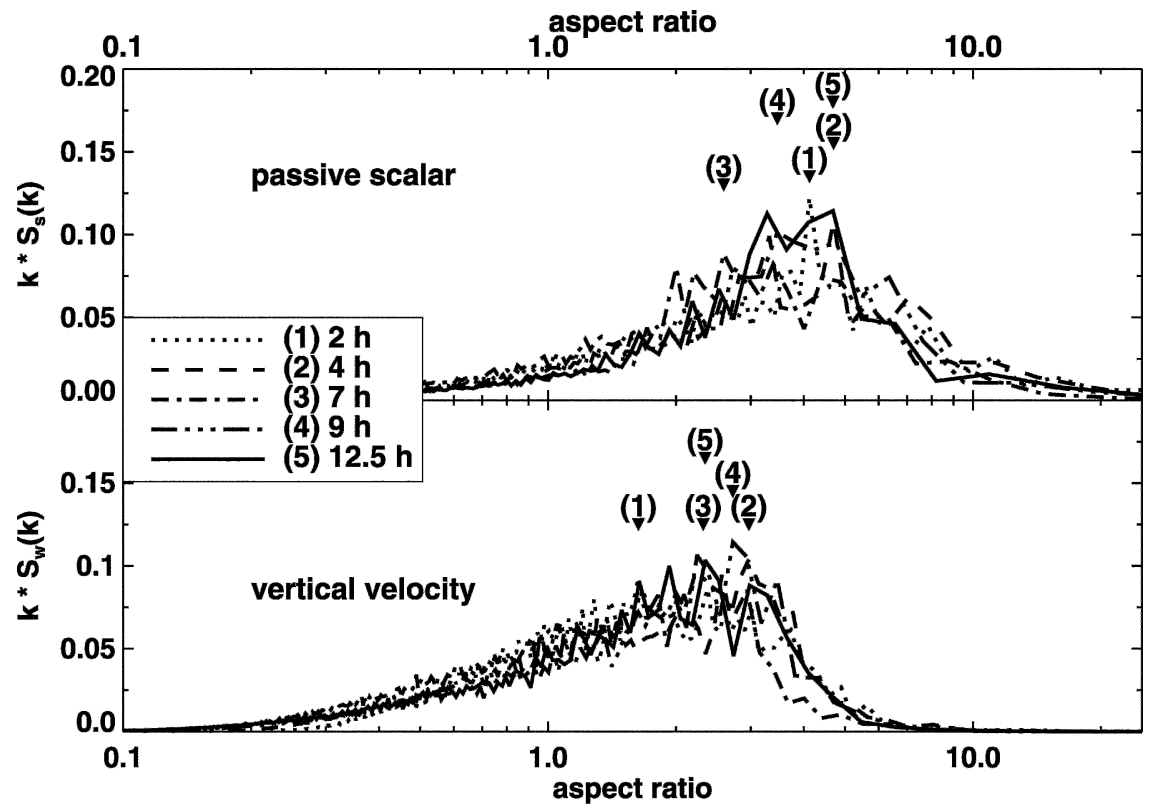

FIG. 6. (top) Power spectra of the passive scalar and (bottom) the vertical velocity, DRY simulation, resulting from two-dimensional Fourier analysis of horizontal cross sections located approximately in the middle of the developing cloud layer. 
(a)
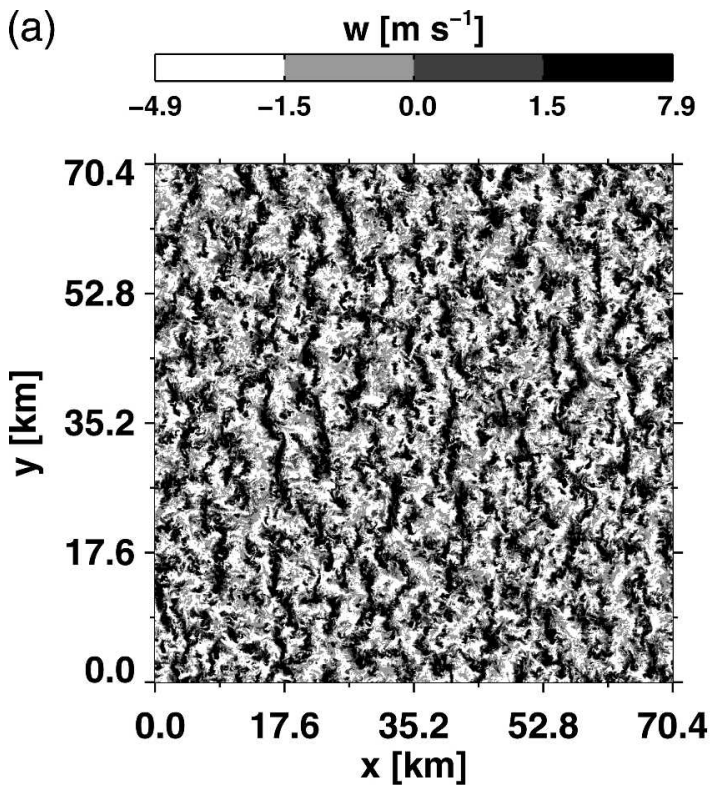

(b)
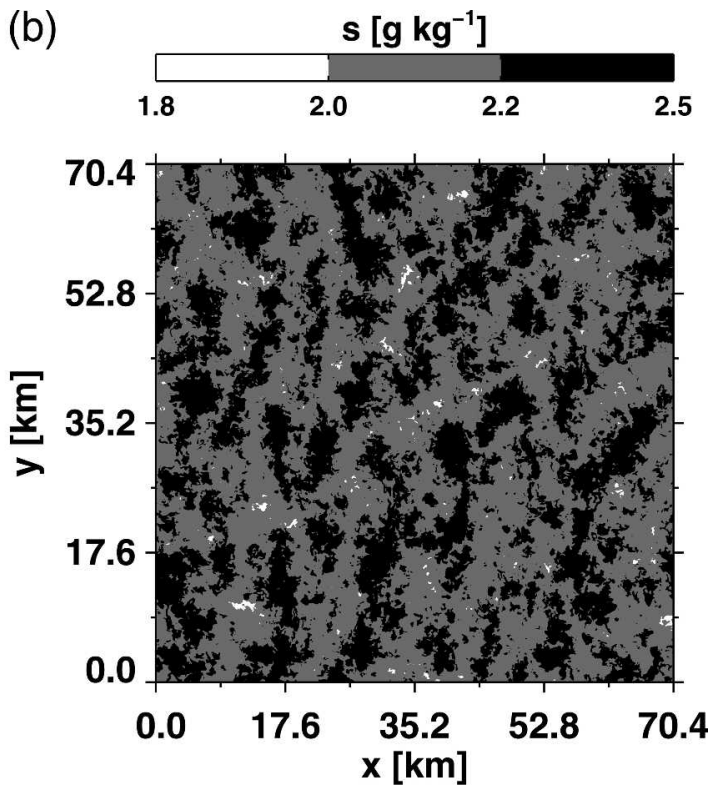

FIG. 7. Horizontal cross section (left) of the vertical velocity $w$ and (right) of the scalar quantity at $t=12.5 \mathrm{~h}$ (DRY simulation). The cross section is located at the level where the resolved variance of the vertical velocity reaches a local maximum $(z=1000 \mathrm{~m})$.

observed. But in contrast to what actually has been observed in the case of the passive scalar, having a flux ratio of 0.2 to 0.3 , increasing scales could be expected. However, since Jonker et al. (1999) as well as de Roode et al. (2004) prescribed small surface heat fluxes and simulated a flow with weak mean wind conditions, we attribute the differences between our DRY case and their CBL simulation to the different boundary conditions.

MC96 proposed a conceptual model to explain the process of cell broadening due to cloud-top cooling. They state that an additional cooling at the cloud top produces colder downdrafts. As a consequence of colder downdrafts, the aspect ratios should increase because they increase the horizontal distance that has to be passed by the (near surface) horizontally outflowing air parcels before they become positively buoyant and form an updraft again. However, we believe that this argument is not convincing because an increasing temperature difference between the sea and the overlying air locally increases the surface heat flux so that cooler air receives more energy than comparably warmer air reducing its time to become positively buoyant. Our doubt of the conceptual model of MC96 is supported by the results of WET. Here, intensive small-scaled regions of positive and negative temperature fluctuations are randomly distributed throughout the whole nearsurface layer as could be seen by the correlation functions (Fig. 5). At regions of warmer and colder air, small-scaled up- and downdrafts occur, respectively. Hence, near the surface the flow structure is characterized by small scales and not, as the conceptual model of MC96 implies, by large scales.
Currently, we are investigating the hypothesis of Dörnbrack (1997) using passive particle advection to answer questions about mechanisms of cell broadening. Dörnbrack (1997) hypothesizes that the additional cooling at the cloud top and the evaporation processes lead to an increase of the effective horizontal diffusion, which possibly yields the broadening of convective cells. The assumption of Dörnbrack (1997) is based on the observation that the total kinetic energy of the flow is increased by additional diabatic heat sources.

Acknowledgments. This investigation was supported by the Deutsche Forschungsgemeinschaft under contract numbers RA 617/3-3 and JA 1115/1-1. All runs were performed on the IBM Regatta at the HLRN and on 256 processing elements of the Cray-T3ELC384 of the Konrad-Zuse-Zentrum für Informationstechnik in Berlin (ZIB). For the WET simulation on the CrayT3E, each processing element required $115 \mathrm{CPU}$ h (4.8 days) in total. We appreciate the permission to include a figure from Müller and Chlond (1996). The critical review by an anonymous reviewer and Bjorn Stevens greatly improved the manuscript. Paquita Zuidema helped in calculating cloud reflectivities from LWP fields.

\section{REFERENCES}

Agee, E. M., 1984: Observations from space and thermal convection: A historical perspective. Bull. Amer. Meteor. Soc., 65, 938-949.

Atkinson, B. W., and J. W. Zhang, 1996: Mesoscale shallow convection in the atmosphere. Rev. Geophys., 34, 403-431.

Bretherton, C. S., and M. C. Wyant, 1997: Moisture transport, 
lower-tropospheric stability, and decoupling of cloud-topped boundary layers. J. Atmos. Sci., 54, 148-167.

Brümmer, B., 1992: ARKTIS 1991, -Report on the field phase with examples of measurements. Berichte aus dem Zentrum für Meeres- und Klimaforschung, Reihe A Nr. 3, Zentrum für Meeres- und Klimaforschung. [Available from Meteorologisches Institut, Bundesstr. 55, Hamburg D-20146, Germany.]

_- 1999: Roll and cell convection in wintertime arctic cold-air outbreak. J. Atmos. Sci., 56, 2613-2636.

— cellular structures during KonTur. Beitr. Phys. Atmos., 59, $162-184$.

Cuijpers, J. W. M., and P. G. Duynkerke, 1993: Large eddy simulation of trade wind cumulus clouds. J. Atmos. Sci., 50, 38943908.

de Roode, S. R., P. G. Duynkerke, and H. J. J. Jonker, 2004: Large-eddy simulation: How large is large enough? J. Atmos. Sci., 61, 403-421.

Dörnbrack, A., 1997: Broadening of convective cells. Quart. J. Roy. Meteor. Soc., 123, 829-847.

Etling, D., and R. A. Brown, 1993: Roll vortices in the planetary boundary layer: A review. Bound.-Layer Meteor., 65, 215248.

Fiedler, B. H., 1993: Cell broadening in three-dimensional thermal convection between poorly conducting boundaries. Beitr. Phys. Atmos., 66, 173-181.

— dimensional thermal convection between poorly conducting boundaries: Large eddy simulation. Beitr. Phys. Atmos., 3, 235-241.

Hardy, K. R., and H. Ottersten, 1969: Radar investigations of convective patterns in the clear atmosphere. J. Atmos. Sci., 26, 666-672.

Jonker, H. J. J., P. G. Duynkerke, and J. W. M. Cuipers, 1999:
Mesoscale fluctuations in scalars generated by boundary layer convection. J. Atmos. Sci., 56, 801-808.

Kanda, M., A. Inagaki, M. O. Letzel, S. Raasch, and T. Watanabe, 2004: LES study on the energy imbalance problem with eddy covariance fluxes. Bound.-Layer Meteor., 110, 381-404.

Letzel, M. O., and S. Raasch, 2003: Large-eddy simulations of thermally induced oscillations in the convective boundary layer. J. Atmos. Sci., 60, 2328-2341.

Moeng, C.-H., and J. C. Wyngaard, 1988: Spectral analysis of large-eddy simulations of the convective boundary layer. $J$. Atmos. Sci., 45, 3573-3587.

Müller, G., and A. Chlond, 1996: Three-dimensional numerical study of cell broadening during cold-air outbreaks. Bound.Layer Meteor., 81, 289-323.

Noh, Y., W.-G. Cheon, S.-Y. Hong, and S. Raasch, 2003: Improvement of the K-profile model for the planetary boundary layer based on large eddy simulation data. Bound.-Layer Meteor., 107, 401-427.

Raasch, S., and M. Schröter, 2001: PALM-A large-eddy simulation model performing on massively parallel computers. $\mathrm{Me}$ teor. Z., 10, 363-372.

Ray, D., 1965: Cellular convection with nonisotropic eddys. Tellus, 17, 434-439.

Slingo, A., 1989: A GCM parameterization for the shortwave radiative properties of water clouds. J. Atmos. Sci., 46, 14191427.

Stevens, B., 2000: Cloud transitions and decoupling in shea-free stratocumulus-topped boundary layers. Geophys. Res. Lett., 27, 2557-2560.

Turton, J. D., and S. Nicholls, 1987: A study of the diurnal variation of stratocumulus using a multiple mixed layer model. Quart. J. Roy. Meteor. Soc., 113, 969-1009.

Weinbrecht, S., and S. Raasch, 2001: High resolution simulations of the turbulent flow in the vicinity of an Arctic lead. $J$. Geophys. Res., 106, 27 035-27046. 